VOL. $2(1970), 247-252$.

\title{
One variable equations over semigroups
}

\section{Frank Levin}

\begin{abstract}
An analogue of the theorem on the existence of a primitive element for separable extensions of fields is presented for semigroups. This has two immediate consequences.

(i) A semigroup is algebraically closed with respect to equations in several variables if and only if it is closed with respect to equations in a single variable.

(ii) Any countable semigroup $C$ is embedded in a two-generator semigroup, one of whose generators is in $C$.
\end{abstract}

Further, a proof is given that any free product of a semigroup of order one with one of order two is $S Q$-universal, that is, its factor semigroups embed all countable semigroups. The proofs are adaptations of one used by Trevor Evans, Proc. Amer. Math. Soc. 3 (1952), 614-620, to show that a free product of two infinite cyclic semigroups is $S Q$-universal.

The theorem on the existence of a primitive element for fields states that for separable extensions of fields the adjunction of several algebraic elements to a field can be accomplished with a single adjunction. An analogous result holds for groups (see [3]). The following theorem extends this to semigroups and thus answers a question posed by Professor B.H. Neumann in connection with investigations into algebraically closed semigroups.

THEOREM 1. Let $A$ be a semigroup containing a subsemigroup $B$

Received 10 January 1970. The author gratefully acknowledges support by the National Science Foundation. 
such that $A$ can be generated by $B$ and a countable subset $a_{1}, a_{2}, \ldots$, of eilements of $A$. Then there exists on oversemigroup $A_{1}$ of $A$ and an element $a \in A_{1}$ such that $A_{1}$ is generated by $B$ and the single (primitive) element $a$. Further, to any prescribed integers $n>m \geq 3$, the oversemigroup $A_{1}$ can be so chosen that the primitive element $a \in A_{1}$ satisfies $a^{n}=a^{m}$.

The proof of Theorem 1 is essentially a variation of one used by Trevor Evans in [2] to show that any countable semigroup can be embedded in a two-generator semigroup. Before demonstrating the proof, however, we first list two immediate consequences of the Theorem.

The first application of the Theorem is to equations over semigroups. An equation over a semigroup $S$ has the form

(1) $f\left(x_{1}, x_{2}, \ldots, x_{n} ; s_{1}, s_{2}, \ldots\right)=g\left(x_{1}, x_{2}, \ldots, x_{n} ; s_{1}, s_{2}, \ldots\right)$

where $f$ and $g$ are words in the variables $x_{i}$ and the elements $s_{i} \in S .(1)$ is consistent over $S$ if $S$ can be embedded in an oversemigroup $T$ containing elements $t_{i}$ such that ( $I$ ) is satisfied in $T$ with $x_{i}=t_{i} \cdot S$ is $(m, n)$-algebraically closed if every consistent system of $m$ equations in $n$ variables can be solved in $S$ itself. The following Corollary then follows directly from the Theorem.

COROLLARY 1. S is $(m, n)$-algebraically closed if and only if it is $(m, 1)$-algebraically closed.

The second application of the theorem extends Evans' result.

COROLLARY 2. Let $C$ be a countable semigroup. Then $C$ can be embedded in a two-generator semigroup generated by any arbitrarily chosen element $c \in C$ and a second generator $g$ which satisfies, for any prescribed integers $n>m \geq 3$, the relation $g^{n}=g^{m}$.

The second Corollary follows from the Theorem by taking $B$ as the cyclic semigroup generated by $c$. In particular, if $C$ contains a zero element $O$, then $c$ can be chosen as 0 . Otherwise, we may first embed $C$ in the semigroup obtained from $C$ by adjoining a zero element.

The proof of the Theorem is based on the following Lemma. Unless 
otherwise specified we use the notation of Clifford and Preston [1]. In particular, $\Delta S$ denotes the diagonal of $S \times S$, and if $S$ is any semigroup without identity element, then $S^{l}$ denotes the semigroup obtained from $S$ by adding an identity element 1 to $S$. Otherwise, $S^{1}=S$. Also, $\left\langle x ; x^{m}=x^{n}\right\rangle$ denotes the cyclic semigroup generated by $x$ satisfying $x^{m}=x^{n}$, and $S * T$ denotes the free product of $S$ and $T$.

LEMMA. Let $S$ and $T$ be semigroups and $P=S * T$. Let $Q$ be a subsemigroup of $P$ having the following properties:

(i) the subsemigroup $H$ of $P$ generated by $S$ and $Q$ is their free product;

(ii) $u v \in H, u \in S$ imply that $v \in H$;

(iii) upv $\in H, p \in H \backslash S, u, v \in P^{l}$ imply that $u, v \in H^{1}$.

Let $\beta$ be a congruence on $H$ such that $\beta \cap(S \times S)=\Delta S$ and $\alpha$ be the congruence on $P$ generated by $\beta$. Then $P / \alpha$ embeds $H / \beta$ naturally and $\alpha \cap(S \times S)=\Delta S$.

Proof. By Lemma 9.9 of [1], $H / \beta$ is naturally embedded in $P / \alpha$ if and only if $\alpha \cap(H \times H)=\beta$. Thus, to prove the Lemma we must consider elements of the form

$$
\left(w_{1}, w_{2}\right)=\left(u_{0} p_{1} u_{1} p_{2} \ldots p_{k} u_{k}, u_{0} q_{1} u_{1} q_{2} \ldots q_{k} u_{k}\right)
$$

where for each $i,\left(p_{i}, q_{i}\right) \in B, u_{i} \in P^{l}$, and show that $\left(w_{1}, w_{2}\right) \in H \times H$ implies $\left(w_{1}, w_{2}\right) \in \beta$.

The proof is by induction on $k$. First, let $\left(u_{0} p_{1} u_{1}, u_{0} q_{1} u_{1}\right) \in(H \times H)$. Since $B \cap(S \times S)=\Delta S$ we may assume $\left(p_{1}, q_{1}\right) \notin S \times S$. In particular, suppose $p_{1} \notin S$. Then, by (iii), $u_{0} p_{1} u_{1} \in H$ implies that $u_{0}, u_{1} \in H^{1}$ so that $\left(u_{0} p_{1} u_{1}, u_{0} q_{1} u_{1}\right) \in \beta$. In the general case (2), we may again assume $p_{1} \in H \backslash S$. Then by (iii), $u_{0}, u_{1} p_{2} \ldots p_{k} u_{k} \in H$. Since $u_{0} q_{1} \in H$ we have, by (ii) or (iii), whichever is applicable, that $u_{1} q_{2} \ldots q_{k} u_{k} \in H$ so that $\left(u_{1} p_{2} \ldots p_{k} u_{k}, u_{1} q_{2} \ldots q_{k} u_{k}\right) \in(H \times H) \cap \alpha$. By induction, it now follows 
that $u_{i} \in H$ for all $i$ so that $\left(w_{1}, w_{2}\right) \in \beta$, which proves the Lema since $\alpha \cap(S \times S)=\beta \cap(S \times S)=\Delta S$.

Proof of Theorem 1. Let $P=B *\langle x\rangle$ and $b \in B$ be an arbitrarily chosen element of $B$. We define elements $h_{i} \in P$ in one-to-one correspondence with the generators $a_{i}$ of $A$ as follows:

$$
h_{i}=x b x^{2+i} b x, \quad i=1,2, \ldots .
$$

Let $Q$ be the subsemigroup of $P$ generated by the $h_{i}$. Since an element of $P$ is in $Q$ if and only if it has the form $x b \ldots b x^{2} b x^{2+i} b x^{2} b x^{2+j} b x \ldots b x$, it is clear that $Q$ is freely generated by the $h_{i}$. A similar analysis shows that $H$, the subsemigroup of $P$ generated by $Q$ and $B$ is their free product. Thus, $H$ satisfies (i) of the Lemma with $S$ replaced by $B$. Part (ii) of the Lemma is trivial to verify, and ( $i$ ii) follows from the special forms of the elements of $H \backslash B$. Thus, the Lemma applies. In particular, since $H=B * Q$ there is a congruence $\beta$ on $H$ with $B \cap(B \times B)=\Delta B$ and $H / B \cong A$. (That is, $H$ can be mapped homomorphically onto $A$ by $\varphi$, say, where $\varphi$ is the identity map on $B$ and maps each $h_{i}$ onto $a_{i} \in A$. ) Thus, if $\alpha$ is the congruence on $P$ generated by $\beta$, then $P / \alpha$ embeds $H / \beta \cong A$ naturally and since $\alpha \cap(B \times B)=\Delta B, B$ is embedded in $P / \alpha$, which proves the first part of the Theorem since $P / \alpha$ is generated by $B \alpha$ and $x \alpha$.

For the second part of the Theorem we further embed the oversemigroup $A_{1}=P / \alpha$ constructed above. Thus, consider $A_{1}$, a semigroup generated by $B$ and $a \in A_{1}$. Let now $P_{1}=B *\left\langle y ; y^{n}=y^{m}\right), n>m \geq 3$. Choose $b \in B$ as above and set $h=y b y^{3} b y$. Clearly, $h$ generates an infinite cyclic subsemigroup $Q_{1} \subset P_{1}$. Let $H_{1}$ be generated in $P_{1}$ by $B$ and $Q_{1}$. As above, we obtain that $H_{1}=B * Q_{1}$ so that $H_{1}$ satisfies (i) of the Lemma. Part ( $i i$ ) of the Lerma is also easy to verify here. For Part (iii), we need only remark that since $n>m \geq 3$, the relation for $y$ has no effect in deciding if an element lies in $H_{l}$ since products of elements of $H_{1}$ introduce, at most, quadratic factors of $y$. Thus, the Lemma is again applicable, and since $H_{1}=B * Q_{1}$ there is, as above, a congruence 
$\beta_{1}$ on $H_{1}$ such that $H_{1} / \beta_{1} \cong A_{1}$ and $\beta_{1} \cap(B \times B)=\Delta B$. Hence, if $\alpha_{1}$ is the congruence on $P_{1}$,generated by $\beta_{1}$, then $P_{1} / \alpha_{1}$ embeds $H_{1} / \beta_{1}$. Finally, since $\alpha_{1} \cap(B \times B)=\Delta B$, we have embedded $A_{1}$ and, hence, $A$ in $P_{1} / \alpha_{1}$ which is generated by $B$ and the congruence class containing $y$, which proves the Theorem.

If we drop the condition in Corollary 2 that one of the two generators of the oversemigroup of $C$ is in $C$, then we obtain a slightly sharper result. For the following Theorem let $P_{2}=\left\langle x ; x^{2}=x\right\rangle * T_{2}$, where $T_{2}$ is any semigroup of order 2 with elements $t_{1}, t_{2}$, say.

THEOREM 2. Let $C$ be a countabie semigroup. Then $C$ can be embedded in a factor semigroup of $P_{2}$.

Proof. First embed $C$ in a two-generator semigroup $C_{1}$ as provided by Corollary 2 or Evans [2]. Let $H_{2} \subset P_{2}$ be the subsemigroup of $P_{2}$ generated by $h_{1}=x t_{1} x$ and $h_{2}=x t_{2} x$. An element $h \in H_{2}$ has the form $h=x s_{1} x s_{2} \ldots s_{k} x$, where $s_{i} \in T_{2}$. Since $h$ is uniquely determined by the sequence $s_{1}, s_{2}, \ldots, s_{k}$, it is clear that $H_{2}$ is freely generated by the $h_{i}$. Thus, there is a congruence $\beta_{2}$ on $H_{2}$ such that $H_{2} / \beta_{2}=C_{1}$. Let $\alpha_{2}$ be the congruence of $P_{2}$ generated by $\beta_{2}$. Again, the proof will be completed by showing that $P_{2} / \alpha_{2}$ embeds $\mathrm{H}_{2} / \beta_{2}$ or, equivalently, that $\alpha_{2} \cap\left(H_{2} \times H_{2}\right)=\beta_{2}$. Thus, let $\left(p_{i}, q_{i}\right) \in \beta_{2}, i=1,2, \ldots$, and

$$
\left(w_{1}, w_{2}\right)=\left(u_{0} p_{1} u_{1} p_{2} \ldots p_{k} u_{k}, u_{0} q_{1} u_{1} q_{2} \ldots q_{k} u_{k}\right) \in H_{2} \times H_{2},
$$

where $u_{i} \in P_{2}^{1}$. Since $x^{2}=x$ and each $p_{i}$ and $q_{i}$ begins and ends with an $x,\left(w_{1}, w_{2}\right)$ can be expressed in the form

$$
\left(w_{1}, w_{2}\right)=\left(v_{o} p_{1} v_{1} p_{2} \ldots p_{k} v_{k}, v_{o} q_{1} v_{1} q_{2} \ldots q_{k} v_{k}\right)
$$

where, for each $i, v_{i}=x u_{i} x$ if $u_{i} \notin H_{2}^{1}$ and $v_{i}=u_{i}$, otherwise. In either case each $v_{i} \in H_{2}^{1}$ so that $\left(w_{1}, w_{2}\right) \in B_{2}$, which proves the Theorem. 


\section{References}

[1] A.H. Clifford and G.8. Preston, The aZgebraic theory of semigroups, Vol. Il (Math. Surveys 7 (II), Amer. Math. Soc., Providence, Rhode Island, 1967).

[2] Trevor Evans, "Embedding theorems for multiplicative systems and projective geometries", Proc. Amer. Math. Soc. 3 (1952), 614-620.

[3] Frank Levin, "One variable equations over groups", Arch. Math. 15 (1964), 179-188.

Rutgers, The State University,

New Brunswick, New Jersey, USA. 